REGARDS

SUR L'ECONOMIE ALLEMAND

BULLETIN ECONOMIQUE DUCIRAC
Regards sur l'économie allemande

Bulletin économique du CIRAC

$82 \mid 2007$

Varia

\title{
La croissance tient bon
}

Isabelle Bourgeois

\section{(2) OpenEdition}

Journals

Édition électronique

URL : http://journals.openedition.org/rea/635

DOI : 10.4000/rea.635

ISBN : 978-2-8218-0860-7

ISSN : 1965-0787

Éditeur

CIRAC

Édition imprimée

Date de publication : 1 juillet 2007

Pagination : 3-4

ISSN : 1156-8992

Référence électronique

Isabelle Bourgeois, "La croissance tient bon », Regards sur l'économie allemande [En ligne], 82 | juillet 2007, mis en ligne le 30 avril 2008, consulté le 15 septembre 2020. URL : http://

journals.openedition.org/rea/635 


\section{La croissance tient bon}

Le moral des Allemands est au beau fixe. Les uns après les autres, experts et instituts de conjoncture revoient à la hausse leurs prévisions de croissance pour l'année en cours. Alors que dans leur rapport conjoint de printemps, les six instituts avançaient une hausse du PIB de $2,4 \%$, les estimations sont maintenant plus près de la barre des $3 \%$, voire la dépassent comme dans le cas de l'IfW de Kiel. Les baromètres mesurant la confiance des ménages ou le climat des affaires traduisent eux aussi un solide optimisme. En effet, contrairement à ce que pensaient les conjoncturistes voici peu encore, "l'évolution de l'économie allemande est assise sur des bases beaucoup plus larges qu'on imaginait », affirme l'institut ZEW (Mannheim) dans son analyse conjoncturelle de juin. En effet, les trois moteurs de la croissance - exportations, investissements et consommation - tournent à plein.

$\mathrm{Si}$, au cours du premier trimestre, la croissance a légèrement fléchi, avec $+0,5 \%$ seulement en données CVS (contre $+1 \%$ au trimestre précédent), cela est dû « à une série de facteurs exceptionnels », explique la Bundesbank dans son rapport de juin. L'un d'entre eux a été la hausse de la TVA qui a artificiellement gonflé la croissance des derniers mois de 2006, la tassant en retour au début de 2007. Mais cela n'a pas modifié la tendance de fond : d'octobre 2006 à mars 2007 en comparaison des six mois précédents, le PIB a crû de $+3,3 \%$, et «la tendance cyclique devrait rester orientée à la hausse », souligne la même Bundesbank dans son rapport de mai dont l'un des chapitres est consacré à l'impact de la hausse de la TVA (une analyse au long cours menée avec Destatis).

Globalement, l'indice du coût de la vie a augmenté de $+1,9 \%$ au premier trimestre. Cependant, il faut distinguer selon ses composantes : l'énergie s'est renchérie de $+2,4 \%$, les biens manufacturés (hors tabac et énergie) ont augmenté de $+1 \%$, voire plus pour l'automobile $(+3,0)$, alors que les prix de l'électroménager et de l'équipement de la maison continuaient de baisser. Les services (hors loyers) se sont renchéris de $+2 \%$, surtout en matière d'assurance $(+2,6 \%)$ ou d'entretien automobile. Mais, ajoute la Buba, les prix de ces biens et services auraient augmenté de toute façon; et elle cite l'exemple de l'introduction, dans une série de Länder, de frais d'inscription à l'université, qui s'est soldée par une hausse de $+25,1 \%$ de l'indice des coûts de formation ; celui-ci 'pèse' 6,66 \% dans l'indice global du coût de la vie. Au mois de mai, ajoute Destatis des son côté, les principaux responsables de la hausse sont l'électricité $(+6,1 \%)$, le gaz $(+3,1 \%)$ et les carburants $(+1,9 \%)$, mais aussi les voyages organisés $(+5,7 \%)$. Cela étant, la hausse est compensée par la baisse des prix du fuel domestique $(-10 \%)$, de l'électronique de loisirs $(-8,8 \%)$ ou des équipements informatiques (-26,8\%). Et si le prix des tomates a baissé de $-21,8 \%$, celui des pommes de terre a, lui, augmenté de $+10 \%$ (et même de $+121 \%$ entre janvier et février, comme se plaît à rappeler la Buba ; outre-Rhin, la pomme de terre est l'équivalent de notre baguette). Au total, en mai, le taux d'inflation s'établissait à $+2 \%$. Il reste difficile, au vu de ces données, de faire la part de la hausse de la TVA, du renchérissement des matières premières ou des effets saisonniers, voire météorologiques...

La consommation, quant à elle, ne s'en est pas réellement ressentie, si on excepte son infléchissement au cours du premier trimestre $(-0,2 \%$ par rapport au premier trimestre 2006), mais après une hausse anticipée à la fin 2006. Les trois points supplémentaires de TVA n'ont pas été répercutés en totalité sur les prix au consommateur, estime l'institut HWWI (Hambourg); sur l'année, l'indice du coût de la vie devrait s'accroître de $+1,8 \%$, et de $+1,5 \%$ seulement en 2008 . Certes, ces points seront progressivement répercutés d'ici la fin de l'année, mais sans grever la consommation des ménages, soutenue par un pouvoir d'achat en hausse.

Car la principale raison du faible impact de la hausse de la TVA réside dans l'accroissement du revenu des ménages. Elle a deux origines. La première est la série de hausses des salaires négociées au printemps dans la chimie, le BTP et l'industrie des métaux, qui se solde par une augmentation de $+0,8 \%$ des salaires conventionnels en un an au total, et même de $+1,8 \%$ en comparaison du dernier trimestre 2006. Ces hausses relativement élevées (+4,1\% dans la métallurgie de juin 2007 à juin 2008 par exemple, suivis d'une progression de $+1,7 \%$ ) « reflètent l'amélioration cyclique de la position des syndicats dans la négociation qui résulte d'un taux d'utilisation des capacités particulièrement favorable et d'un riche stock de commandes », explique la Bundesbank (rapport de mai), avant de poursuivre: "il s'agit aussi d'une participation des salariés au succès économique des entreprises qu'a permis la modération salariale de ces dernières années ».

Autrement dit, indirectement et à son niveau, la fin de la modération salariale confirme la bonne tenue des activités industrielles. Les carnets de commande ont continué de se
Une tendance cyclique orientée à la hausse

L'effet TVA est difficilement mesurable

La hausse n'a guère affecté la consommation des ménages, ...

... soutenue par une hausse des salaires dans l'industrie

Une industrie toujours performante 
Investissements et exportations tirent la croissance..

... et entraînent la création d'emplois remplir au premier trimestre : $+2,5 \%$ par rapport au dernier de 2006 , mais $+13,7 \%$ par rapport au premier trimestre 2007. Les commandes étrangères étaient en hausse (+5\% par rapport à l'hiver), traduisant la demande toujours très forte de biens d'investissement allemands, surtout en construction mécanique, mais aussi en équipements de production et distribution d'électricité. Quant au BTP, désormais assaini, il a bénéficié d'un hiver très doux qui a permis le lancement de nombreux chantiers, ce qui explique l'actuel fléchissement des commandes. Si les chiffres d'avril ont été nettement moins favorables pour la production industrielle (-2,4\% par rapport à mars), il faut en chercher la raison dans les grèves qui ont affecté la métallurgie et, plus encore, dans le 'pont' du 1er mai. L'industrie reste performante, comme le montre la comparaison avec la période mars/ avril de 2006 : en un an, la production a crû de $+7,1 \%$, rappelle le ministère fédéral de l'Economie. Comme le taux d'utilisation des capacités atteint 87,5\%, les entreprises sont tenues d'investir pour répondre à la demande. Le ministère y voit un signe « que la croissance portée par l'investissement se poursuivra » (communiqué du 19-06-07), d'autant qu'elle est soutenue parallèlement par l'optimisme inaltérable des milieux d'affaires.

Car l'investissement est désormais le second moteur de la croissance outre-Rhin, qu'il s'agisse de biens d'équipement (+8,6\% entre les premiers trimestres 2007 et 2006) ou de construction $(+15,3 \%)$. Si la réforme de la fiscalité des entreprises qui entrera en vigueur en 2008 (le régime de l'amortissement dégressif sera supprimé) devrait inciter les entreprises à anticiper l'extension de leurs capacités, la légère baisse des dépenses qui s'ensuivra au début 2008 ne devrait avoir qu'un impact marginal sur l'investissement, tiré avant tout par la demande intérieure et étrangère. Car pour la première fois depuis 2000 , celui-ci est significativement en hausse, comme l'explique la Bundesbank dans son rapport de juin. De 2005 à 2006, le taux d'investissement net est en effet passé de 2,7\% à 3,9\% du revenu disponible (ménages et entreprises). Quant aux exportations, elles continuent et continueront de porter la croissance allemande. Elles se sont certes légèrement ralenties au premier trimestre (-1,2\% par rapport aux mois d'hiver) où leur apport à la croissance a même été négatif (elles l'ont tassée de 2,0 points) mais en partant d'un niveau record: l'excédent commercial allemand était de 164,6 milliards $€$ en 2006. Le ralentissement de la demande américaine devrait être compensé par la dynamique du marché européen et celle des économies émergentes. En avril, la valeur des exportations atteignait 78,8 milliards $€$, soit une hausse de $+13,1 \%$ par rapport à avril 2006 .

La seconde cause de l'optimisme des ménages est l'embellie désormais tangible sur le marché de l'emploi. Au premier trimestre, le nombre d'actifs occupés s'est accru de plus d'un demi-million ; mieux encore, l'amélioration se poursuit depuis l'été 2006, et elle gagne en ampleur. Au point que le nombre de chômeurs devrait passer en dessous de la barre de 3,5 millions en 2008 selon les instituts et l'Agence fédérale pour l'emploi, et le taux de chômage s'établir à 8,7 \% en moyenne sur 2007 avant de tomber à 7,9 \% l'année suivante $(\mathrm{HWWl})$. Vers la fin de l'été, estime l'institut IfW, le nombre de bénéficiaires de l'Arbeitslosengeld I (allocations versées pendant les 12 premiers mois) pourrait être inférieur à 1 million, ce qui devrait porter l'excédent de l'Agence de Nuremberg à 5,5 milliards $€$ et ainsi permettre à terme une réduction de 0,3 point des cotisations chômage. « Les Allemands sont à nouveau persuadés que la croissance s'installe durablement, ce qui les incite à prévoir des achats importants », résume l'institut GfK.

LA CONFIANCE DES ACTEURS ECONOMIQUES est également soutenue par le recul du déficit public qui, du fait de la hausse des recettes fiscales induite par la croissance, devrait tomber à $0,3 \%$ du PIB d'ici la fin de l'année selon l'institut HWWI. Peer Steinbruck, le ministre fédéral des Finances, se montre certes plus prudent, n'avançant que $0,5 \%$ à la fin juin, mais se laisse porter lui aussi par l'optimisme général : au début du mois de juin encore, il annonçait à la Commission un déficit de 1,2\%. L'Allemagne respecte à nouveau l'un des critères du Pacte de stabilité et de croissance ; un léger excédent est même envisageable dès 2008 à en croire l'institut IfW, en 2011 seulement selon le ministère fédéral des Finances. Ensuite, elle pourra engager la réduction de sa dette. C'est en tout cas ce que prévoit le plan budgétaire à moyen terme du Bund que le gouvernement a prévu d'adopter le 4 juillet. Reste d'ici là à juguler les tentations dépensières de certains ministères (28 milliards $€$ au moins d'ici 2011). Bien que le ministre fédéral de l'Economie, Michael Glos, souhaite lancer un programme d'allégements fiscaux et d'investissements (chiffré à 70 milliards $€$ ), il se plie à la discipline budgétaire, résumant ainsi la politique de l'Allemagne lors du Conseil ECOFIN du 5 juin : « il importe de consolider la tendance à la croissance et de mettre à profit cette dynamique pour poursuivre la réduction du déficit... et faire profiter in fine les citoyens de la consolidation budgétaire ».

Isabelle Bourgeois (26-06-2007) 